\title{
Hydrometeorological Observation and Study in High Altitude Area
}

\author{
Yongjian Ding, ${ }^{1}$ Shiqiang Zhang, ${ }^{2}$ Fengjing Liu, ${ }^{3}$ \\ Daqing Yang, ${ }^{4}$ Chansheng $\mathrm{He}^{5}{ }^{5}$ and Svetlana Stuefer ${ }^{6}$ \\ ${ }^{1}$ Cold and Arid Regions Environmental and Engineering Research Institute, Chinese Academy of Sciences, Lanzhou 730000, China \\ ${ }^{2}$ College of Urban and Environmental Sciences, Northwest University, No. 1, Xian 710027, China \\ ${ }^{3}$ Department of Agriculture and Environmental Science and Cooperative Research Programs, Lincoln University, \\ 207 Foster Hall, Jefferson City, MO 65101, USA \\ ${ }^{4}$ National Hydrology Research Centre, University of Saskatchewan, 11 Innovation Boulevard, Saskatoon, SK, Canada S7N $3 H 5$ \\ ${ }^{5}$ Department of Geography, Western Michigan University, Kalamazoo, MI 49008-5424, USA \\ ${ }^{6}$ University of Alaska Fairbanks, AK 99775-5860, USA
}

Correspondence should be addressed to Yongjian Ding; dyj@lzb.ac.cn

Received 8 September 2015; Accepted 10 September 2015

Copyright (C) 2016 Yongjian Ding et al. This is an open access article distributed under the Creative Commons Attribution License, which permits unrestricted use, distribution, and reproduction in any medium, provided the original work is properly cited.

The cryosphere in high altitude area plays a major role in the Earth's system through its impact on the surface energy budget, sea level change, water cycle, primary productivity, and surface gas exchange and is thus a fundamental control on physical, biological, and social environment over substantial areas of the Earth's surface. Knowledge and understanding of all the components of the cryosphere have substantially improved our understanding of the specific processes and their impact on climate, hydrology, and economy.

We invite investigators to contribute original research articles as well as review articles that will stimulate the continuing efforts to improve the technology of hydrometeorological observation by different means in high altitude area, the water-heat process in glaciers, snow, or permafrost, the understanding of water cycle in high altitude area, the climate change in high altitude area, and the impact of climate change on hydrological process and water resource in high altitude areas. The accepted papers focus on these topics.

Spatial distribution of precipitation is very complex, and generally very limited observed precipitations are available in high altitude areas. Estimation of the spatial and temporal distribution of precipitation is very important for hydrological analysis and modeling. Included in this special issue is one paper on this aspect. The paper evaluated the accuracy of different precipitation datasets over Tianshan Mountainous Area by intensified observation data and gives one good example on the method of how to integrate the remote precipitation dataset with the limited observation data.

Glaciers are very sensitive to climate change. Study on glacier change and the albedo on glacier surface will help us to advance understanding of the mechanism of glacier response to climate change. Included in this special issue are two papers on this aspect. One paper estimates the glacier area change and the driving factors in Ili river basin. Another paper analyzed the variation of glacier surface albedo and effecting factors on Dongkemadi glacier. Both papers improve our understanding about the glacier change mechanism in high altitude regions.

Ecosystems in high altitudes are most sensitive and influential to climate change. Process-based studies will help us understand not only how ecosystems respond to climate change but also how climate change is driven by changes in ecosystems. Included in this special issue are two papers on this aspect. One was to understand hydrochemical denudation and transient carbon dioxide drawdown in highly glacierized areas in the Tianshan Mountains, China, and the other was to understand the role of mountain groundwater in hydrologic cycle in the Qilian Mountains, China. Both papers 
help improve our understanding of how glaciation affects/is affected by climate change and how groundwater meditates river runoff under a changing climate.

Snow sublimation and grassland evapotranspiration are very important process in high altitude area. The complexities of modelling and measuring sublimation and evapotranspiration limit investigations to smaller scales in complex terrain in previous studies. Included in this special issue are two papers on the aspect. One paper reviews the effects that microscale controls have on sublimation, and the vegetation cover variability in macroscale was considered to be the most important factor to advance understanding of snow sublimation, which improve our understanding of the snow sublimation process in macroscale and how to simulate the sublimation process. Another paper tried to estimate the grassland evapotranspiration at field scale by using different method, which gives interesting results. Both papers help improve our understanding of the evaporation process in high altitude regions.

Water storage in basin scale provides one new clue to the validation of the water balance in inland river basin, where main water resource developed in high altitude regions. Included in this special issue is one paper on the aspect. The paper evaluated the water storage change of inland cryosphere in western China, which improves our understanding on the water balance in western China, where many inland river basins are included.

Yongjian Ding

Shiqiang Zhang Fengjing Liu

Daqing Yang

Chansheng He

Svetlana Stuefer 

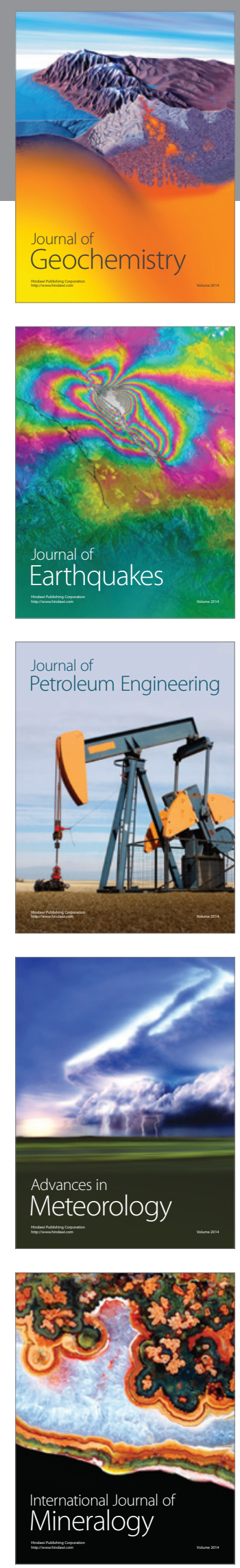
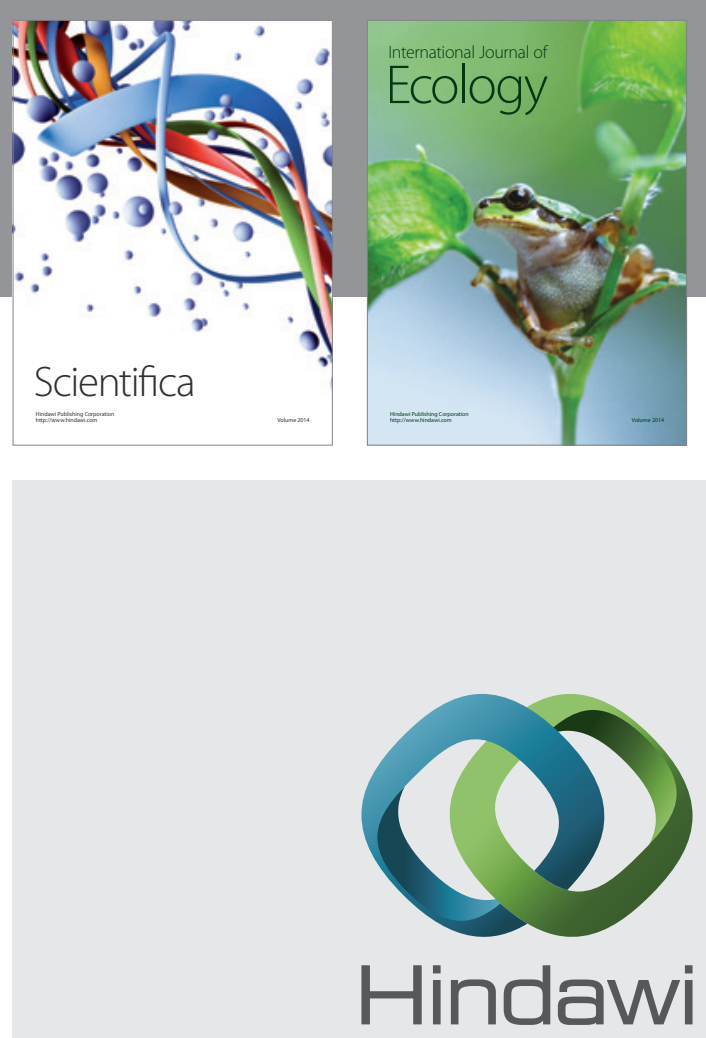

Submit your manuscripts at

http://www.hindawi.com
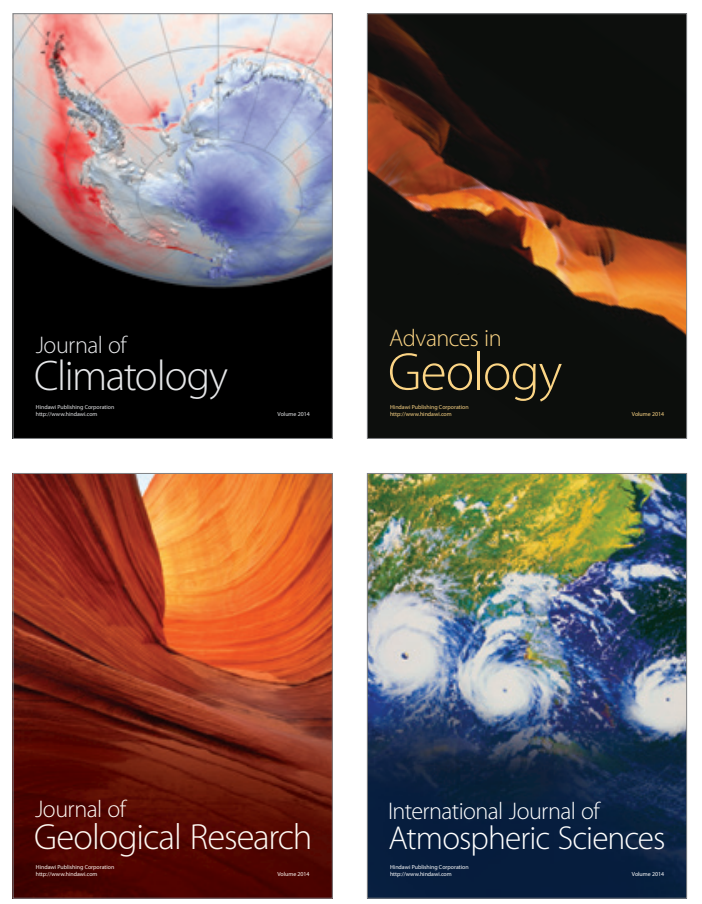

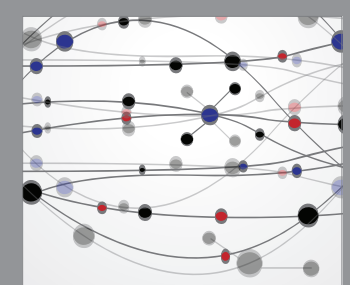

The Scientific

\section{World Journal}
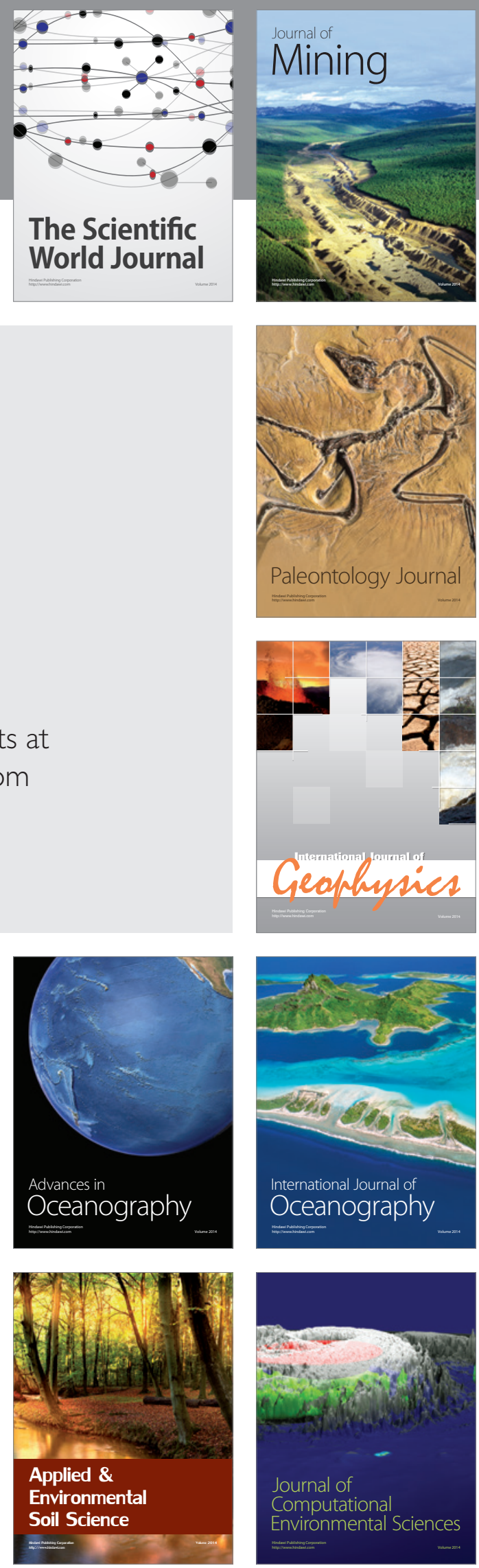